\title{
Modified Panax ginseng extract regulates autophagy by AMPK signaling in A549 human lung cancer cells
}

\author{
HWA-SEUNG YOO $^{1 *}$, JUNG MIN KIM ${ }^{2 *}$, EUNBI JO ${ }^{3 *}$, CHONG-KWAN CHO $^{1}$, SEUNG-YEUL LEE ${ }^{3}$, \\ HWAN SU KANG ${ }^{3}$, MIN-GOO LEE ${ }^{4}$, PEI-YING YANG ${ }^{5}$ and IK-SOON JANG ${ }^{3}$ \\ ${ }^{1}$ East-West Cancer Center, Dunsan Oriental Hospital of Daejeon University, Daejeon 302-122; \\ ${ }^{2}$ NAR Center, Inc. \& Genoplan Korea, Inc., Seoul 06221; ${ }^{3}$ Division of Bioconvergence Analysis, \\ Korea Basic Science Institute, Daejeon 305-333; ${ }^{4}$ Department of Physiology, Korea University College of Medicine, \\ Seoul 02841, Republic of Korea; ${ }^{5}$ Departments of Palliative, Rehabilitation and Integrative Medicine, \\ The University of Texas MD Anderson Cancer Center, Houston, TX 77030, USA
}

Received August 4, 2016; Accepted October 17, 2016

DOI: 10.3892/or.2017.5590

\begin{abstract}
Panax ginseng has been used worldwide as a traditional medicine for the treatment of cancer and other diseases. The antiproliferative activity of ginseng has been increased after enzymatic processing of ginseng saponin, which may result in the accumulation of minor saponins, such as Rh2, $\mathrm{Rg} 3$, compound $\mathrm{K}$ and protopanaxatriol type (PPT) in modified regular ginseng extract (MRGX). In the present study, the anticancer activity and the associated mechanisms of MRGX were investigated using A549 human lung cancer cells. To elucidate the mechanisms underlying the effects of MRGX, we performed a microarray analysis of gene expression in the A549 cells. Molecular mechanisms that were associated with the anticancer activity of MRGX were studied, with a special focus on the autophagy-related multiple signaling pathways in lung cancer cells. Microarray analyses elucidated autophagyrelated genes affected by MRGX. Administration of MRGX at $100 \mu \mathrm{g} / \mathrm{ml}$ induced punctate cytoplasmic expression of LC3, Beclin-1 and ATG5 and increased expression of endogenous LC3-II whereas $50 \mu \mathrm{g} / \mathrm{ml}$ did not inhibit the proliferation of
\end{abstract}

Correspondence to: Dr Ik-Soon Jang, Drug and Disease Target Group, Division of Bioconvergence Analysis, Korea Basic Science Institute, Gwahangno 113, Yuseong-gu, Daejeon 305-333, Republic of Korea

E-mail: jangiksn@kbsi.re.kr

Dr Pei-Ying Yang, Departments of Palliative, Rehabilitation and Integrative Medicine, Unit 462, The University of Texas MD Anderson Cancer Center, 1515 Holcombe Boulevard, Houston, TX 77030, USA

E-mail: pyang@mdanderson.org

*Contributed equally

Key words: autophagy, AMPK pathway, NSCLC cells, Akt/mTOR pathway
A549 cells. Compared to the control cells, in cells treated with MRGX at $100 \mu \mathrm{g} / \mathrm{ml}$, the level of p-Akt was increased, while that of mTOR-4EBP1 was decreased. Downregulation of mTOR and 4EBP1 in the MRGX-treated cells was found not to be a p-Ulk (S757)-dependent pathway, but a p-Ulk (S317)dependent autophagic pathway, using AMPK. These data suggest that MRGX regulates AMPK and induces autophagy in lung cancer cells.

\section{Introduction}

Panax ginseng C.A. Meyer ( $P$. ginseng) has been used worldwide as a traditional medicine for the treatment of cancer, including lung cancer and other diseases. Among the varieties of ginseng used in medical treatment, $P$. ginseng (Korean ginseng) and $P$. quinquefolius (American ginseng) have been the ones most studied to date $(1,2)$. We found that the anticancer effect of ginseng increased after enzymatic processing of ginseng saponin (3), which may be the result of the accumulation of minor saponins, such as Rh1, Rg3, compound $\mathrm{K}$ and protopanaxatriol type (PPT) constituents in ginseng saponin. The modified regular ginseng extract (MRGX), which reinforces various ginsenosides from regular ginseng butanol-extract (GBX) by treating them with the enzymes laminarinase and pectinase to produce fortified anticancer ginsenosides, is reported to have anticancer effects against lung cancer cells (3-5). In addition, the chemical profiles of enzymatically modified ginseng extract were addressed by high-performance liquid chromatography (HPLC) compared to butanol extract as previously reported by us. Five ginsenosides, including Rh1, Rg3(S), Rg3(R), compound K and Rh2, were exclusively detected (6).

Lung cancer, which consists mostly of non-small cell lung cancer (NSCLC) in up to $85 \%$ of the total cases, is the second leading cause of cancer-related death worldwide (7), and is considered to be an extremely lethal form of cancer (8). Over the past several decades, surgical and combined therapies have contributed to reducing the mortality and the morbidity associated with lung cancer; however, these approaches still have limitations. Complementary and alternative therapy 
using herbal medicine, particularly using gensenosides have recently received increased attention in the Western medical society $(2,9,10)$. Yet, studies concerning their mechanism of action at the molecular biological level are lacking.

Autophagy, a catabolic process for the degradation and recycling of proteins and organelles, is one type of cell death $(11,12)$. It is initiated by the formation of autophagosomes, which are double-membrane vesicles that fuse to lysosomes to form autolysosomes, where the sequestered cellular components are digested by lysosomal enzymes. The autophagic process is fundamental in ridding the cell of aged and aggregated proteins and is the only known mechanism for the disposal of damaged organelles under normal conditions and for disposal of cells under stress. This process is regulated by autophagy factors such as ULK1, ATG13, ATG7, ATG5 and Beclin-1 $(13,14)$. In addition, autophagy occurs as a result of inappropriate cellular signal transduction through the AMPK-linked mammalian target of rapamycin (mTOR). Generally, mTOR is considered a therapeutic target for lung cancer since mTOR is a major pathway that coordinates proper cell growth and proliferation by regulating ribosomal biogenesis and protein translation (15).

In the present study, MRGX was investigated to determine its potential for use as a therapeutic agent for the treatment of lung cancer. The antiproliferative effect of MRGX was assessed in human non-small cell lung adenocarcinoma cells. Additionally, the molecular mechanisms underlying the anticancer activity of MRGX were studied, with a special focus on the autophagy-related multiple signaling pathways in this particular lung cancer type.

\section{Materials and methods}

Preparation of the MRGX extract. The root of regular ginseng (4 years old) was purchased from the National Agricultural Cooperative Foundation (Chuncheongnam-do, Korea). A total of $20 \mathrm{~g}$ of pulverized ginseng root powder was suspended in $380 \mathrm{ml}$ of distilled water and sterilized at $121^{\circ} \mathrm{C}$ for $15 \mathrm{~min}$. The extract was fractionated via extraction with various solvents, including water, methanol and butanol. Ginseng butanolic extract (GBX) was used as a control for regular ginseng. An aliquot of sterilized laminarinase and pectinase (1:1, specific activity units) was added to the suspension, which was then incubated at $40^{\circ} \mathrm{C}$ for 2 days, after which it was evaporated to powder at $60^{\circ} \mathrm{C}$. This enzyme-modified ginseng powder was suspended in $400 \mathrm{ml}$ of $80 \%$ (v/v) methanol. The suspension was sonicated and filtered through Whatman no. 2 filter paper (Whatman International Ltd., Maidstone, UK). The filtrates were combined and evaporated to dryness at $50^{\circ} \mathrm{C}$. The extract was dissolved in $200 \mathrm{ml}$ distilled water, then separated by a funnel and extracted with $200 \mathrm{ml}$ of butanol. The extract was dissolved to a concentration of $10 \%(w / v)$ in $70 \%$ ethanol. A total of 7 ginsenosides, including Rg1, Re, Rf, Rb1, Rc, Rb2 and F1, were present in the GBX. By contrast, 5 ginsenosides, including Rh1, Rg3(S), Rg3(R), compound $\mathrm{K}$ and Rh2, were exclusively detected in MRGX $(6,10)$. The final obtained sample source was named MRGX in the present study. This sample was deposited (BP1234034, http://biorp.kribb.re.kr) in the Biological Resource Center (BRC) in Korea Research Institute of Bioscience and Biotechnology (KRIBB).
Cell lines. Human non-small cell lung cancer cell lines (A549, H596 and H1299) were obtained from the American Type Culture Collection (ATCC; Rockville, MD, USA). Cells were grown in Dulbecco's modified Eagle's medium (DMEM), supplemented with $10 \%$ (v/v) fetal bovine serum (FBS) and 1\% $(\mathrm{w} / \mathrm{v})$ penicillin-streptomycin (both from Gibco, Grand Island, $\mathrm{NY}$, USA), at $37^{\circ} \mathrm{C}$ with $5 \%$ (v/v) $\mathrm{CO}_{2}$.

Cell viability assay. Cells (5x103/well) were placed in a 96-well plate. After a 24-h incubation, the cells were treated with 0,25 , 50 or $100 \mu \mathrm{g} / \mathrm{ml}$ of MRGX for $72 \mathrm{~h}$. Cell viability assays were performed following the procedures published in a previous study (16). In brief, at the end of the treatment, $10 \mu \mathrm{l}$ of Cell Counting Kit-8 solution (Dojindo, Kumamoto, Japan) was added to the cell solution and incubated for $1 \mathrm{~h}$ at $36^{\circ} \mathrm{C}$. In addition, the cells were pretreated with $100 \mathrm{nM}$ of the autophagy inhibitor bafilomycin A1 (BafA1; Sigma-Aldrich, St. Louis, MO, USA), and treated with MRGX at the same concentrations. Cell viability was determined using a microplate reader (Sunrise, Tecan, Switzerland) to measure the absorbance at $450 \mathrm{~nm}$. The assays were performed in triplicate.

Cell cycle analysis with propidium iodide staining. The cell cycle was analyzed using propidium iodide (PI) staining kits (Roche Diagnosis GmbH, Mannheim, Germany) following the instructions provided by the manufacturer. In brief, the cells were incubated with the extract for $48 \mathrm{~h}$, trypsinized, washed twice with phosphate-buffered solution (PBS), and centrifuged at $2,000 \mathrm{rpm}$ for $5 \mathrm{~min}$ at $4^{\circ} \mathrm{C}$. Then, the cells were incubated with $1.4 \mathrm{mg} / \mathrm{ml}$ of PI for $15 \mathrm{~min}$ at room temperature. Measurements were carried out on a flow cytometer (MoFlo Astrios flow cytometer; Beckman-Coulter, Indianapolis, IN, USA) with a 670-nm high-pass filter for PI detection. The data were analyzed using Summit V6.0 software.

Microarray analysis. Total RNA was extracted from vehicleor $100 \mu \mathrm{g} / \mathrm{ml}$ MRGX-treated A549 lung cancer cells. The total RNA from each sample was extracted using TRIzol reagent (Gibco-BRL, Rockville, MD, USA) according to the manufacturer's instructions. For microarray analysis of the MRGX-treated lung cancer cells, the Human Twin Chip ${ }^{\mathrm{TM}}$ Human 44K (Genocheck, Seoul, Korea) was used for the transcription profiling analysis. The microarray analysis was performed following the procedures published in a previous study (3). In brief, the Cy3- (vehicle) and Cy5-labeled (MRGX-treated) cRNAs were hybridized with the Human 44K microarray, and the hybridization images were analyzed with an Agilent DNA Microarray Scanner. All data were normalization and selection of upregulated and downregulated genes was performed using GeneSpring GX 7.3 (Agilent Technologies, Inc., Santa Clara, CA, USA).

Ontology-related network analysis. To study the biological functions of ontology-related regulated genes and proteins through their interaction network, we conducted a bioinformatic network analysis using an Ingenuity Pathway Analysis (IPA; http://www.ingenuity.com). The IPA identifies a gene interaction network on the basis of a regularly updated 'Ingenuity Pathways Knowledge-base'. The updatable database was retrieved from the biological literature. Network 
generation was optimized from the inputted expression profile when possible and was aimed at the production of highly connected networks.

Acridine orange staining for autophagy. Autophagy is characterized by increased formation of acidic vesicular organelles (AVOs; lysosomes and autophagolysosomes), which can be quantified using flow cytometry after staining the cells with acridine orange (AO). AO is a weak base that accumulates in AVOs. The AVOs can be quantified based on the fact that the increase in the intensity of the red fluorescence is proportional to the degree of acidity (17). For the FACS analysis, A549 cells $\left(5 \times 10^{6}\right)$ were placed in a $100-\mathrm{mm}$ culture dish and treated with 0,50 or $100 \mu \mathrm{g} / \mathrm{ml}$ of MRGX. After a 48 -h incubation, $1 \mu \mathrm{g} / \mathrm{ml}$ of $\mathrm{AO}$ was added, and the cells were incubated for $15 \mathrm{~min}$ at $37^{\circ} \mathrm{C}$, and then subjected to flow cytometry examination.

Identification of autophagy using immunofluorescence and confocal microscopy. Human full-length LC3 (microtubuleassociated protein 1 light chain $3 \alpha$ ), Beclin-1 and ATG5 (autophagy-related gene 5, a marker of autophagy), and complementary DNA cloned into pCMV-SPORT6 vectors (LC3, hMU000295; Beclin-1, hMU003282; and ATG5, KU032585) were purchased from the 21 Century Frontier Human Gene Bank (Daejeon, Korea), along with a cloning green fluorescent protein (GFP) sequence. A549 cells were seeded at $5 \times 10^{4}$ cells/well on the coverslip of an 8 -well plate. After $24 \mathrm{~h}$, the cells were transfected using the HilyMax transfection reagent (Dojindo, Kumamoto, Japan) with GFP-LC3, GFP-Beclin-1 and GFP-ATG5 vector for $48 \mathrm{~h}$. After MRGX treatment, cells were washed twice with PBS, fixed with $4 \%(\mathrm{v} / \mathrm{v})$ formaldehyde for $15 \mathrm{~min}$, and then permeabilized with cell permeabilization reagents (FIX \& PERM Cell Permeabilization reagents; Invitrogen, Carlsbad, CA, USA). They were further blocked with $10 \%(\mathrm{w} / \mathrm{v})$ goat serum for $1 \mathrm{~h}$, followed by staining with 4',6-diamidine-2'-phenylindole dihydrochloride (DAPI) solution. For immunostaining, cells were incubated in primary antibody (LC3, Beclin-1, ATG5; Abcam, Eugane, CA, USA) diluted to 1:100 in 10\% (w/v) goat serum in PBS overnight at $4^{\circ} \mathrm{C}$. After the cells had been washed 3 times for 5 min each with PBS, they were incubated in the dark for $1 \mathrm{~h}$ with a 1:1,000 dilution of Alexa Fluor 488-conjugated secondary antibody (Invitrogen). Finally, the slides were washed 3 times with PBS and mounted in mounting medium (Vector, Burlingame, CA, USA). Images were captured using a laser-scanning confocal microscope (LSM 710; Carl Zeiss, Jena, Germany) equipped with a C-Apochromat 40x/1.2 water immersion lens (48-nm argon laser/505- to 550-nm detection range). Final image data were analyzed using ZEN 2009 Light Edition software.

Western blot analysis. The expression levels of autophagyrelated signaling proteins in the cells treated with MRGX and compound C (5 $\mu \mathrm{mol} / \mathrm{l}$; CAS 866405-64-3; SigmaAldrich) were examined using western blotting, as previously described (18). In brief, $30 \mu \mathrm{g}$ of the denatured protein was separated using $12 \%$ polyacrylamide gel electrophoresis and transferred onto a nitrocellulose membrane. The nitrocellulose membrane was then stained with Ponceau $\mathrm{S}$ to position the proteins. The blotted membrane was blocked for $1 \mathrm{~h}$ with $5 \%$ (w/v) skimmed milk in Tween-20 and Tris-buffered saline (TTBS), followed by incubation with a dilution of primary antibodies, LC3-I, p62, Akt, p-Akt, AMPK, p-AMPK, mTOR, p-mTOR, 4EBP1, p-4EBP1, Ulk1, p-Ulk1 (S317) and GAPDH (Cell Signaling Technology, Danvers, MA, USA), at room temperature for $2 \mathrm{~h}$ or at $4^{\circ} \mathrm{C}$ overnight. The membrane was washed 3 times for 5 min each time with $0.1 \%$ (v/v) Tween-20 in TBS before incubation with horseradish-peroxidase (HRP)conjugated goat anti-mouse IgG or HRP-conjugated rabbit anti-goat IgG with a 1:2,000 dilution in TBS containing $5 \%(\mathrm{w} / \mathrm{v})$ skimmed milk at room temperature for $1 \mathrm{~h}$. The membrane was rinsed 3 times with TBS-0.1\% (v/v) Tween-20 for 5 min each time and an enhanced chemiluminescence system (Thermo Scientific, San Jose, CA, USA) was used to visualize the bands on a ChemiDoc MP system (Bio-Rad, Hercules, CA, USA). The expression levels of the proteins were quantitatively analyzed by comparison with GAPDH as an internal control.

Statistical analyses. GraphPad Prism software (GraphPad, San Diego, CA, USA) was used for the statistical analyses. The Student's t-test was used to assess the statistical difference between the control and the MRGX-treated groups. P-values $<0.05$ were considered to indicate a statistically significant result.

\section{Results}

Inhibition of the growth of lung cancer cells by MRGX. In order to investigate the effect of MRGX on the growth of various lung cancer cell lines, we directly treated A549, H596 and H1299 cells with MRGX, and evaluated the growth inhibition using cell viability assays. As shown in Fig. 1A, MRGX inhibited the growth of A549 cells after $24 \mathrm{~h}$ of incubation with no statistical significance (Fig. 1A). Treatment with MRGX for 48 and $72 \mathrm{~h}$ markedly and significantly inhibited the proliferation of the A549 cells (Fig. 1B and C). The inhibitory effect of MRGX on the growth of A549 cells was concentration-dependent. Intriguingly, MRGX only exerted moderate inhibitory effect on the growth of H596 and H1299 cells even after the cells had been treated for $72 \mathrm{~h}$ (Fig. 1D).

Cell cycle analysis of A549 cells following treatment with $M R G X$. The effect of MRGX on the cell cycle distribution of A549 cells was evaluated using PI staining and flow cytometry (Fig. 2). The flow cytometric analysis of A549 cells showed an apparent shift from the G1 to the G2/M phase. In A549 cells treated with a vehicle control for $48 \mathrm{~h}, 90.5 \%$ of the cells were in the G1 phase, and $3.7 \%$ were in the G2/M phase (Fig. 2A). In contrast, in the A549 cells treated with $100 \mu \mathrm{g} /$ $\mathrm{ml}$ of MRGX, $68.0 \%$ were in the G1 phase, and $24.1 \%$ were in the $\mathrm{G} 2 / \mathrm{M}$ phase (Fig. 2C), suggesting that MRGX treatment caused the cells to undergo G2/M phase arrest.

Analysis of MRGX-related gene expression in lung cancer cells. To analyze the MRGX-related gene expression in lung cancer cells, we used a cDNA microarray analysis approach. Microarray data were filtered and combined using gene symbols; and then network analyses were performed. The 
A

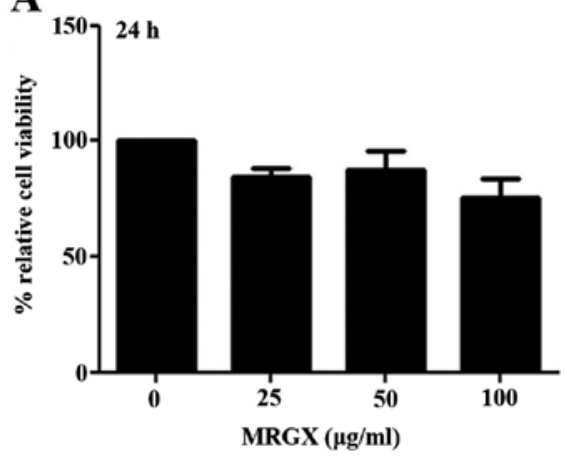

C

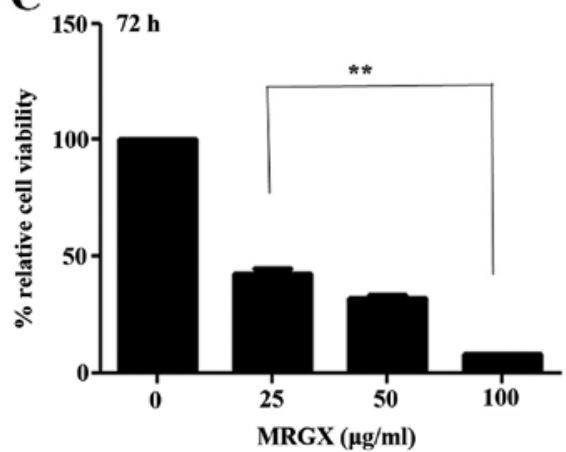

$\mathbf{B}$

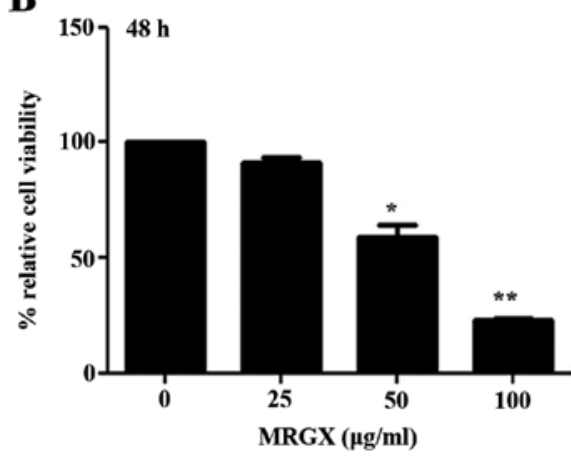

D

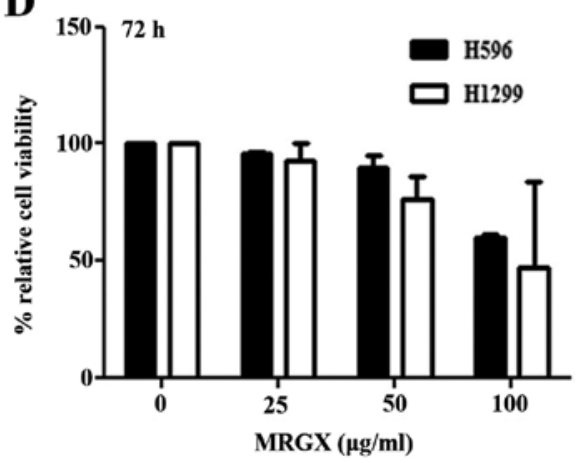

Figure 1. Cell viability assay of lung cancer cells following treatment with MRGX. Lung cancer A549 cells were treated with the indicated concentrations of 0 , 25, 50 or $100 \mu \mathrm{g} / \mathrm{ml}$ of MRGX for (A) 24, (B) 48 and (C) $72 \mathrm{~h}$. (D) Lung cancer H596 and H1299 cells were pretreated with the same concentrations of MRGX for $72 \mathrm{~h}$. The bar graphs present the means \pm SDs from triplicate experiments; ${ }^{*} \mathrm{P}<0.05$ and ${ }^{* *} \mathrm{P}<0.01$ vs. the control.
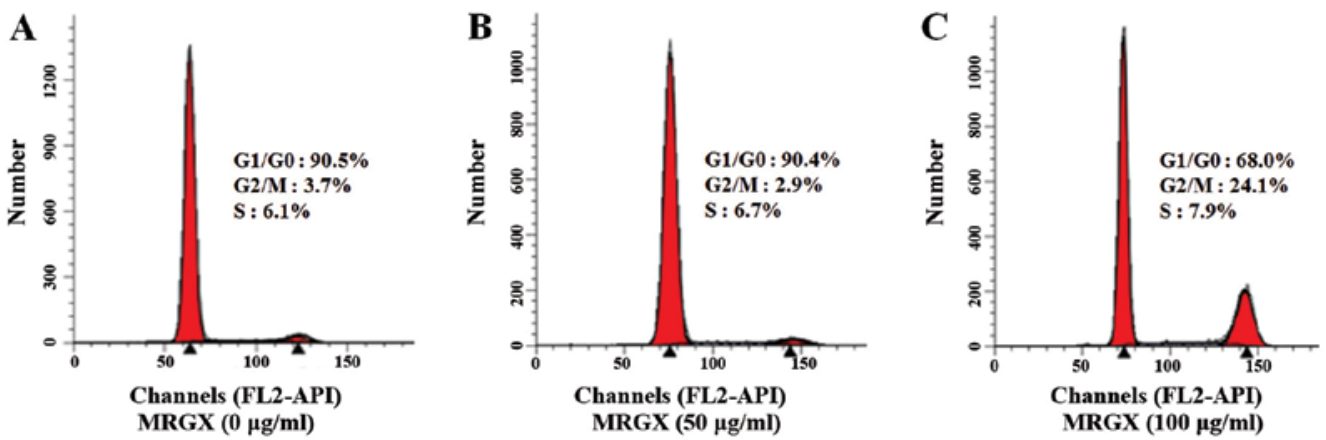

Figure 2. Analysis of cell cycle distribution using flow cytometry in the A549 cells following treatment with MRGX. Dual-parameter flow cytometry was used to quantify the analysis of the MRGX-induced cell cycle in A549 cells. The cells were treated with (A) no MRGX (control), (B) MRGX at $50 \mu \mathrm{g} / \mathrm{ml}$ and (C) MRGX at $100 \mu \mathrm{g} / \mathrm{ml}$ for $48 \mathrm{~h}$. The cells were strained with PI and were then analyzed using flow cytometry. The numbers in the cell cycle curve of each plot indicate the percentages of $\mathrm{G} 1 / \mathrm{G} 0, \mathrm{G} 2 / \mathrm{M}$ and $\mathrm{S}$ phase cells. The data shown are representative of 3 independent experiments.

clustered microarray data showed that groups of genes in the untreated and MRGX-treated $(100 \mu \mathrm{g} / \mathrm{ml})$ lung cancer cells were differentially regulated. Genes were considered to be differentially expressed when the global M-value, $\log _{2}$ (R/G fluorescence), exceeded 1.0 (2-fold-change) with a $\mathrm{P}$-value $<0.05$ after the significance analysis of the microarray (SAM). A total of 1,169 genes were ultimately identified and categorized in terms of their cellular functions using Gene ontology annotation (Fig. 3A). The Venn diagram for autophagy-related increases ( 22 genes) and decreases ( 3 genes) is shown (Fig. 3B). The relative abundances of the regulated genes between the untreated and MRGX-treated lung cancer cells are listed in Table I.
Network analysis based on Gene Ontology analysis. To explore key autophagy proteins related to MRGX treatment in the Gene Ontology analysis of gene functions, we used IPA to query 25 autophagy-related proteins belonging to the proteins upregulated and downregulated by MRGX, resulting in a distinct interconnected network (Fig. 4). Quantitative and qualitative alterations were observed in the MRGX-treated lung cancer cells between the regulation groups. Among them, LC3, ATG5, Beclin-1 and Ulk1 were identified as centers of the MRGX-treated autophagy-related protein network in the lung cancer cells. LC3, a mammalian homolog of yeast Atg8, is a reliable marker of autophagosomes. Tracking the conversion of LC3-I to LC3-II is indicative of autophagic 
A

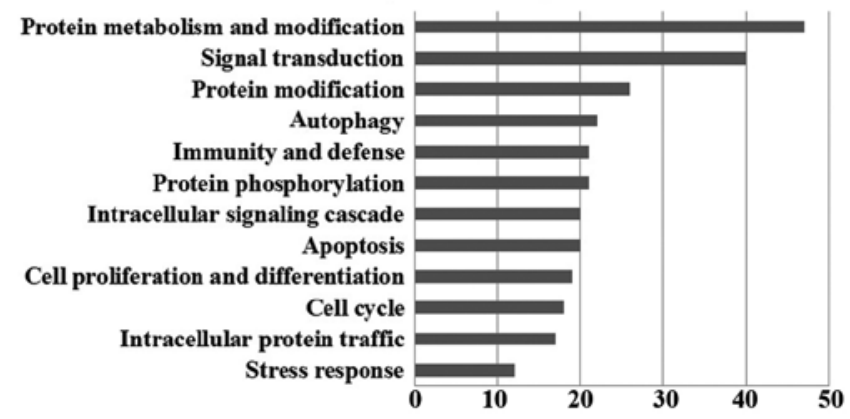

B

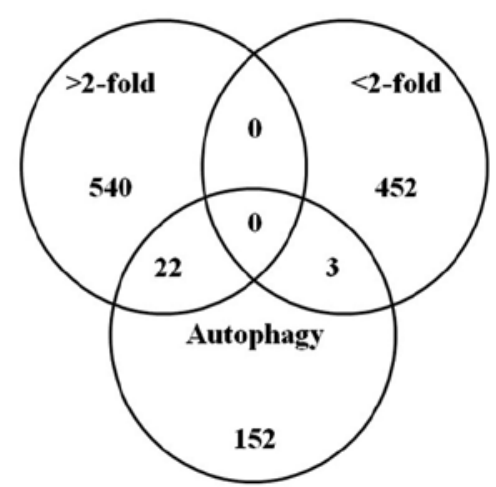

Figure 3. Microarray and Gene Ontology analyses and the autophagic genes whose expression levels were altered following treatment with MRGX. (A) The Gene Ontology analysis revealed differentially expressed genes (>2-fold-regulated genes) in human lung cancer A549 cells in response to MRGX. (B) Gene numbers ( $>2$ - and $<2$-fold, and autophagy-related genes) are shown, and are individually intersected using Venn diagrams.

Table I. Autophagy-related genes affected by MRGX.

\begin{tabular}{|c|c|c|c|c|}
\hline \multirow[b]{2}{*}{ Ratio } & \multicolumn{2}{|c|}{ Signal intensity } & \multirow{2}{*}{$\begin{array}{c}\text { Gene } \\
\text { symbol }\end{array}$} & \multirow{2}{*}{$\begin{array}{c}\text { GenBank } \\
\text { acc. no. }\end{array}$} \\
\hline & MRGX & $\mathrm{Ctl}$ & & \\
\hline 10.66 & 26 & 2 & HSPA9 & NM_004134 \\
\hline 9.82 & 19 & 2 & SMAD2 & NM_001003652 \\
\hline 8.85 & 54 & 6 & BECN1 & NM_003766 \\
\hline 8.21 & 32 & 4 & CTNNB1 & NM_001098209 \\
\hline 7.84 & 60 & 8 & AIFM1 & NM_001130846 \\
\hline 4.53 & 23 & 5 & CLTC & NM_004859 \\
\hline 4.51 & 17 & 4 & CD46 & NM_002389 \\
\hline 4.30 & 23 & 5 & ATG12 & NM_004707 \\
\hline 4.24 & 254 & 60 & SUMO1 & NM_001005781 \\
\hline 4.10 & 38 & 9 & PIK3C3 & NM_002647 \\
\hline 4.05 & 14 & 3 & HIF1A & NM_001530 \\
\hline 3.81 & 27 & 7 & MFN1 & NM_033540 \\
\hline 3.42 & 13 & 4 & XIAP & NM_001167 \\
\hline 3.29 & 266 & 81 & SQSTM1 & NM_001142298 \\
\hline 3.16 & 20 & 6 & PPARG & NM_005037 \\
\hline 3.01 & 75 & 25 & ITGB1 & NM_002211 \\
\hline 2.87 & 280 & 98 & ANXA7 & NM_001156 \\
\hline 2.84 & 420 & 148 & RRAGA & NM_006570 \\
\hline 2.77 & 13 & 5 & PDCD6IP & NM_001162429 \\
\hline 2.62 & 36 & 14 & AURKA & NM_003600 \\
\hline 2.37 & 66 & 28 & ATG3 & NM_022488 \\
\hline 2.22 & 98 & 44 & MAPRE1 & NM_012325 \\
\hline 1.25 & 15 & 12 & ULK1 & NM_003565 \\
\hline 0.43 & 137 & 319 & GSK3A & NM_019884 \\
\hline 0.40 & 141 & 354 & SCRIB & NM_015356 \\
\hline 0.28 & 66 & 239 & TGM2 & NM_004613 \\
\hline
\end{tabular}

The relative expression ratio was calculated by dividing the test channel intensity (MRGX) by the control channel intensity $(\mathrm{Ctl})$.

activity (19). ATG5 is a protein encoded by the ATG5 gene (20). It is an E3 ubiquitin ligase, which is necessary for autophagy due to its role in autophagosome elongation. Beclin-1 is a
Bcl-2-homology (BH)-3 domain only protein. It is expressed in many human tissues and is localized in cytoplasmic structures. Beclin-1 is important for localization of autophagic proteins to a pre-autophagosomal structure (PAS), depending on interactions with the class-III-type phosphoinositide 3-kinase (PI3KC3) (21).

In the present study, Beclin-1 was markedly increased in the MRGX-treated lung cancer cells. The protein-protein network analysis suggests that Beclin-1 is a major protein that interacts with multiple proteins and that it is directly or indirectly downregulated or upregulated in MRGX-treated lung cancer cells. Proteins linked to Beclin-1 include ATG3 and Ulk1. Ulk1 plays a specific role in autophagy in lung cancer (22). However, the detailed mechanism has not yet been studied.

AO staining for autophagy. AO is a versatile fluorescence dye used to stain acidic vacuoles (lysosomes, endosomes and autophagosomes), RNA and DNA in living cells. AVOs were quantified using $\mathrm{AO}$ staining and flow cytometry. In AO-stained cells, the cytoplasm and the nucleolus fluoresce bright green and dark red, respectively, whereas the acidic compartment fluoresces bright red (23). The proportion of AVOs shifted from 1.96 to $9.66 \%$ (Quadrant 3 of Fig. 5A and C) and from 5.44 to $7.33 \%$ (Quadrant 2 of Fig. 5A and C) in the cells treated with $100 \mu \mathrm{g} / \mathrm{ml}$ of MRGX compared with $5.70 \%$ (Quadrant 3 of Fig. 5B) and 10.3\% (Quadrant 2 of Fig. 5B) for cells treated with $50 \mu \mathrm{g} / \mathrm{ml}$ of MRGX. AO-stained A549 cells showed AVOs that had mostly accumulated in the cytoplasm of the cells after having been exposed to $100 \mu \mathrm{g} / \mathrm{ml}$ of MRGX (Fig. 5C).

MRGX treatment induces the autophagy of A549 cells. We screened the A549 cells for autophagy-inducing compounds that stably expressed GFP-LC3, GFP-Beclin-1 and GFP-ATG5 (24-26). When autophagy is induced, GFP-LC3, Beclin-1 and ATG5 are distinctive compared to the control. We treated transfected A549 cells with MRGX and used fluorescent microscopy to examine LC-3, Beclin-1 and ATG5 staining. MRGX (50 and $100 \mu \mathrm{g} / \mathrm{ml}$ ) induced autophagy, as evidenced by the increased and distinctive GFP-LC3 staining in the cytoplasm of the cells treated with MRGX at a concen- 


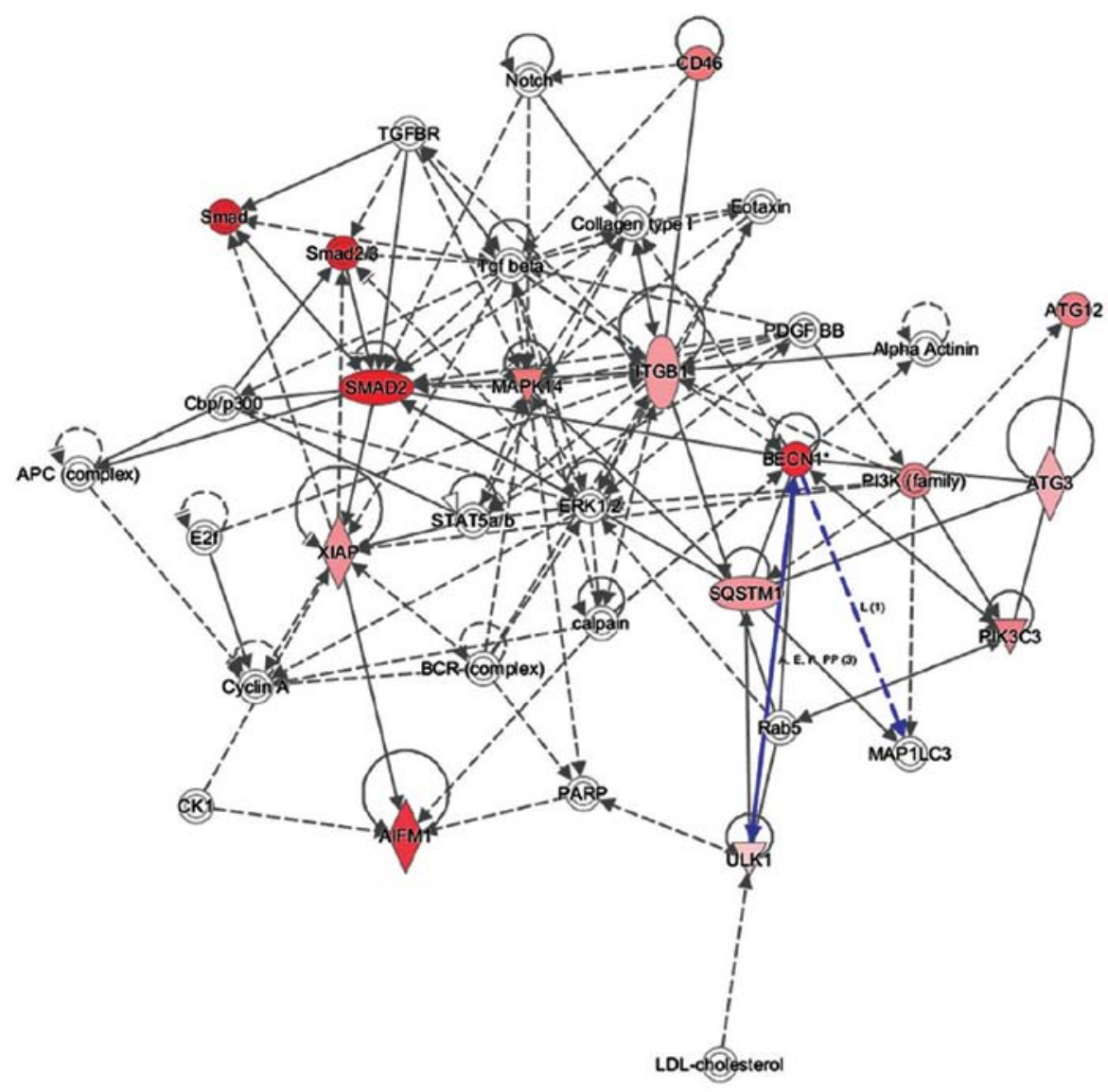

Figure 4. Network analysis based on Gene Ontology data. Proteins whose expression levels were altlered by at least 2-fold in response to MRGX were queried in IPA, which yielded a distinct interconnected network of 25 proteins.
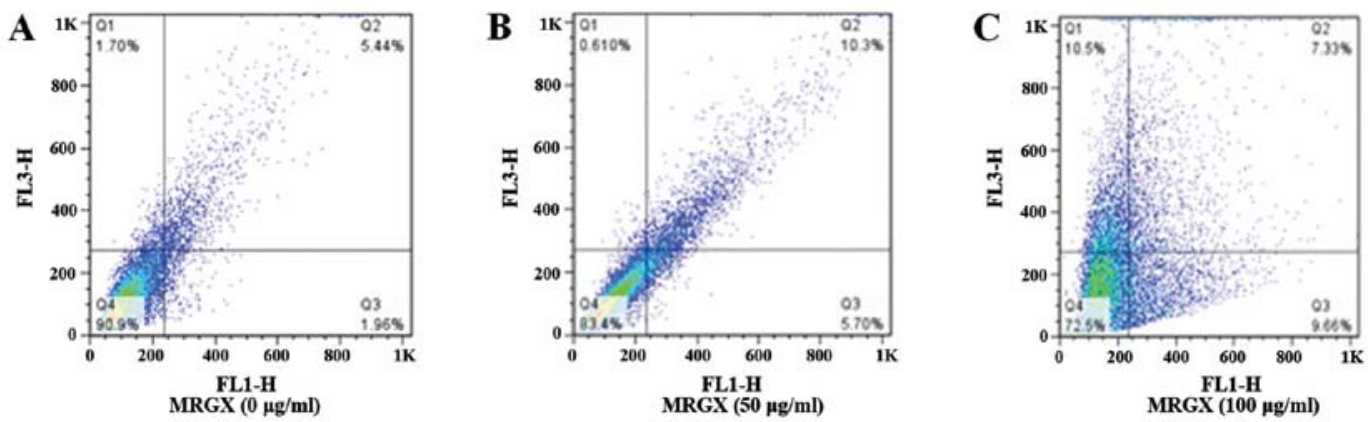

Figure 5. Acridine orange staining for autophagy. Acridine orange staining for autophagy in A549 cells (A) not treated with MRGX (control), and cells treated with MRGX at (B) 50 and (C) $100 \mu \mathrm{g} / \mathrm{ml}$ for $48 \mathrm{~h}$ is shown. The number of AVOs was quantified using acridine orange staining and flow cytometry. The AVOs were observed accumulated in the cytoplasm of cells exposed to 0,50 and $100 \mu \mathrm{g} / \mathrm{ml}$ of MRGX.

tration of $50 \mu \mathrm{g} / \mathrm{ml}$, suggesting that MRGX induces autophagy in a dose-dependent manner (Fig. 6A). In addition, Beclin-1 and ATG5 were stained in the cytoplasm of the cells treated with $50 \mu \mathrm{g} / \mathrm{ml}$ of MRGX (Fig. 6B). The induction of autophagy by MRGX in A549 cells was further tested by examining the expression levels of the autophagy marker proteins LC3, p62, Beclin-1 and ATG5. Similarly, the levels of the LC3-II, Beclin-1 and ATG5 proteins were increased and the level of p62 was decreased in a dose-dependent manner, collectively indicating that MRGX actively triggers autophagy (Fig. 6C). To confirm that MRGX induces autophagy, we analyzed the effect of MRGX-induced autophagy in the A549 cells cultured for $72 \mathrm{~h}$ in the presence of BafA1 (an autophagy inhibitor). The inhibitory effect of BafA1 on MRGX-induced autophagy in the A549 cells was concentration-dependent (Fig. 6D). These data therefore indicated that MRGX induced autophagy of the A549 cells.

$M R G X$ regulates the Ulk-dependent autophagy pathway by $A M P K$. Autophagy in A549 cells is involved in the activation of Akt-mediated mTOR-AMPK-Ulk1 pathways $(27,28)$. To investigate whether MRGX-induced autophagy is mediated through the AMPK-Ulk1 pathway, we compared the expression levels of Akt, mTOR, 4EBP1, AMPK and Ulk1 in A549 cells 
A

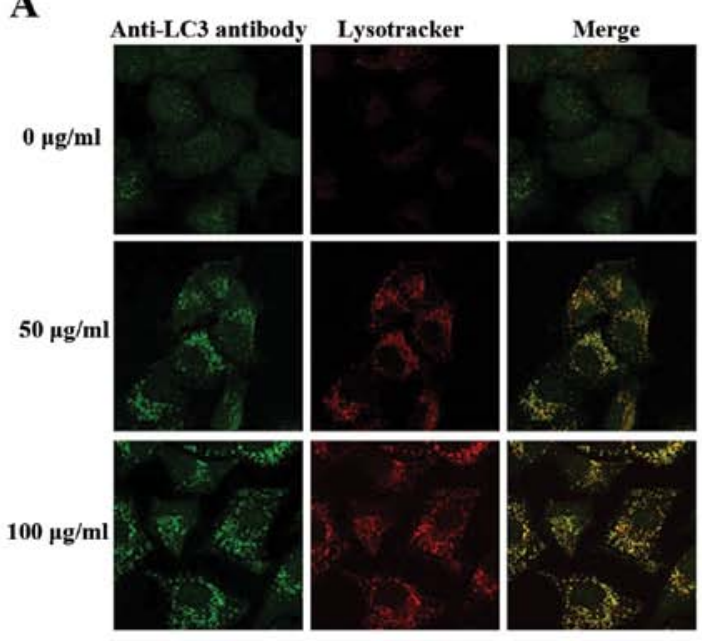

B
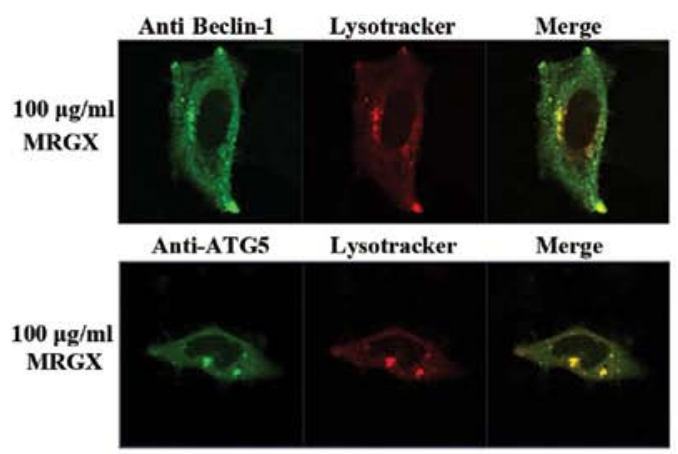

C
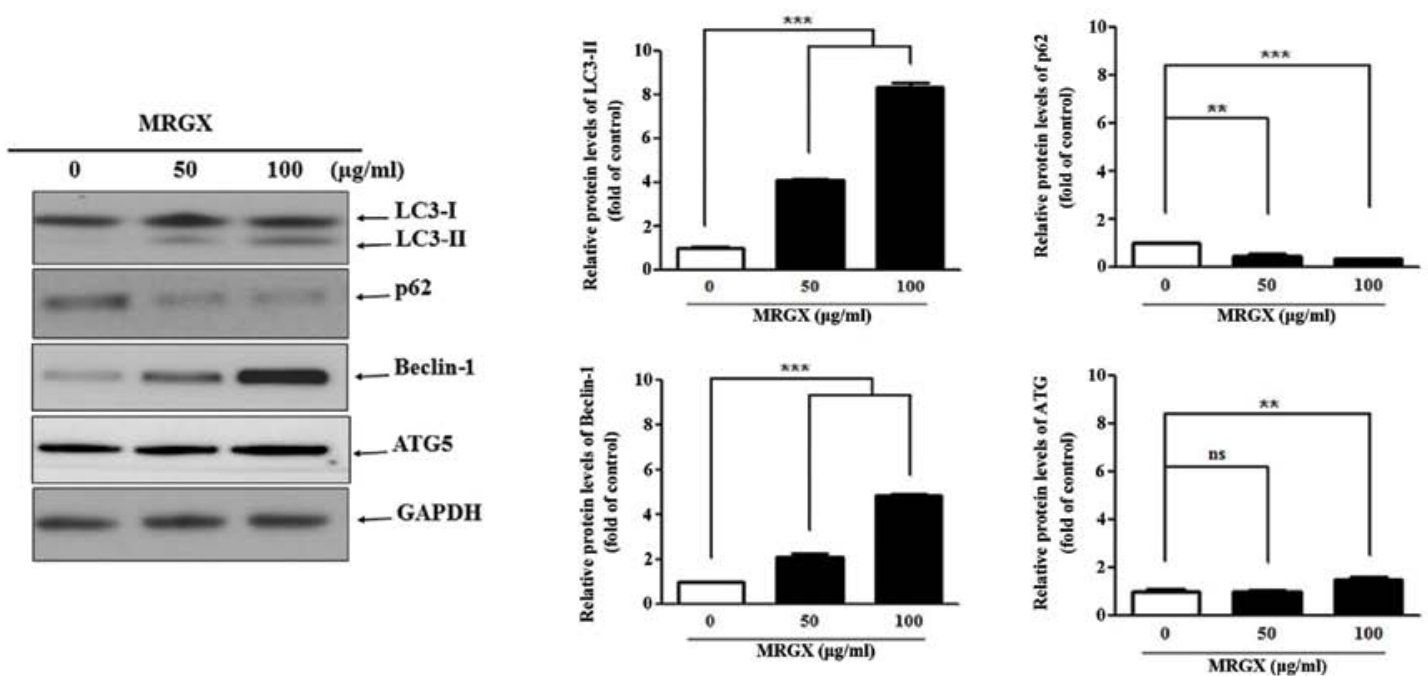

D

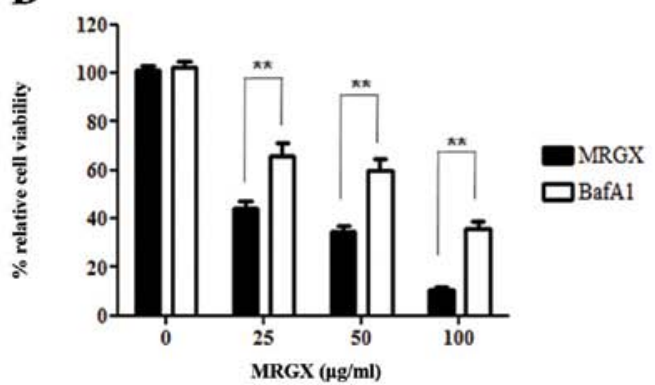

Figure 6. MRGX induces autophagy in A549 cells. (A) A549 cells stably expressing GFP-LC3 (GFP-LC3 cells) were treated with the indicated concentrations $(0,50$ and $100 \mu \mathrm{g} / \mathrm{ml})$ of MRGX for $48 \mathrm{~h}$. Cells were fixed and examined using confocal microscopy. (B) A549 cells stably expressing GFP-Beclin-1 and GFP-ATG5 were treated with the indicated concentration of $50 \mu \mathrm{g} / \mathrm{ml}$ of MRGX for $48 \mathrm{~h}$. Cells were fixed and examined using confocal microscopy. (C) A549 cells were treated with MRGX for $48 \mathrm{~h}$ and the expression of autophagosome markers was examined using western blotting. The levels of LC3-II, Beclin-1 and ATG4 were increased, and the level of p62 was decreased in cells treated with MRGX. Data are expressed as means \pm SD. ${ }^{* *} \mathrm{P}<0.01,{ }^{* * *} \mathrm{P}<0.001$. (D) A549 cells were treated with the indicated concentrations of $0,25,50$ or $100 \mu \mathrm{g} / \mathrm{ml}$ of MRGX with bafilomycin A1 (100 nM) (BafA1, autophagy inhibitor) for $72 \mathrm{~h}$. The bar graphs present the means \pm SDs from triplicate experiments; ${ }^{* *} \mathrm{P}<0.01$ vs. the BafA1-untreated cells; n.s., not significant.

with and without MRGX treatment using western blot analysis. The expression level of phosphorylated AMPK (T172) was increased in the A549 cells treated with $100 \mu \mathrm{g} / \mathrm{ml}$ of MRGX, as was that of p-Akt. p-mTOR and p-4EBP1 expression levels were decreased in the A549 cells treated with 50 and $100 \mu \mathrm{g} / \mathrm{ml}$ of MRGX, suggesting that mTOR signaling was inhibited by MRGX through AMPK-Akt signaling. Ulk1 is a large protein that can be phosphorylated in a serine/threonine-rich region of a molecule such as S317. MRGX increased the phosphorylation of Ulk1 (S317), but not p-Ulk1 (S757) (Fig. 7A). Compound C (dorsomorphin) is an available agent that is used as a cellpermeable AMPK inhibitor. When A549 cells were treated with compound $\mathrm{C}$ together with MRGX, p-AKT and p-AMPK were increased (Fig. 7B). Even though compound $\mathrm{C}$ induces 
A

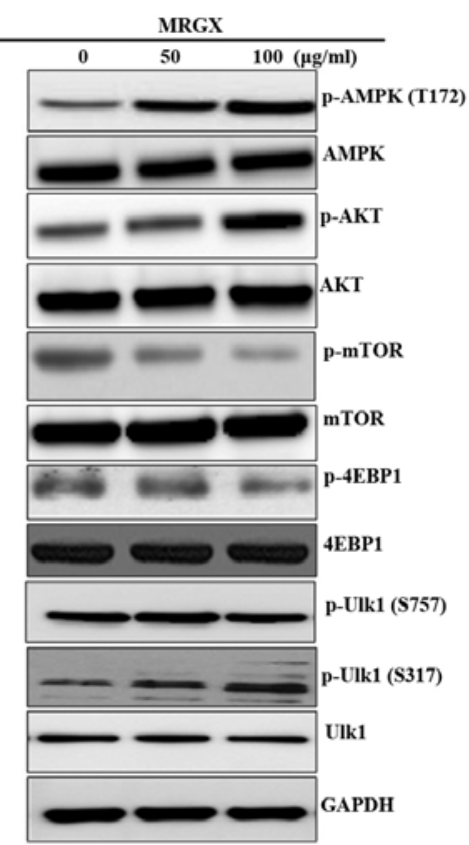

B

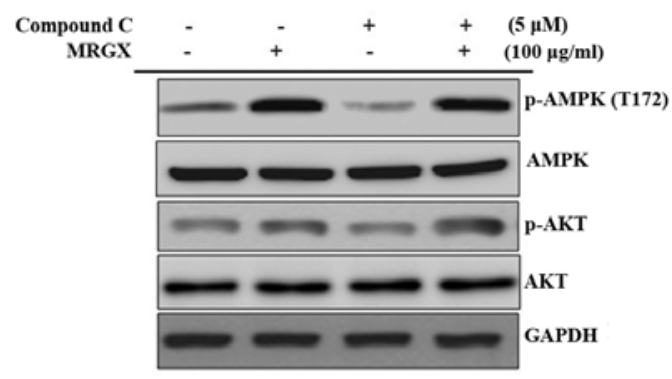

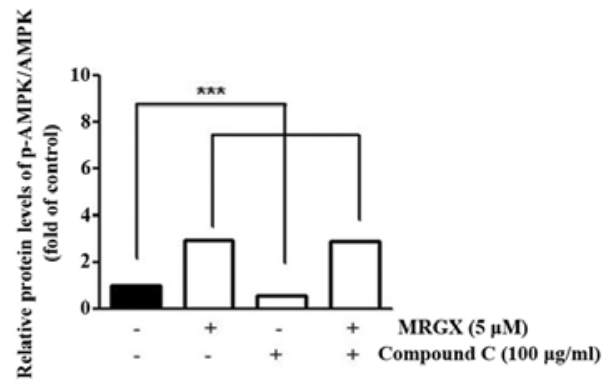

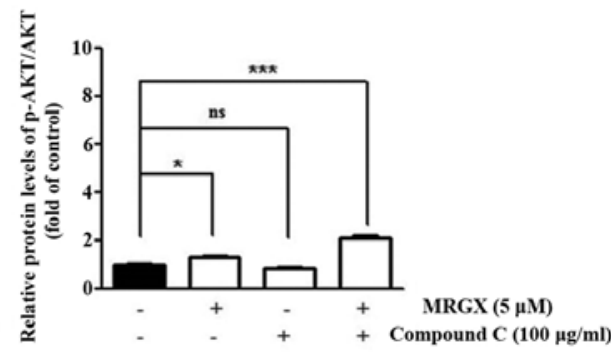

Figure 7. MRGX regulates the Ulk-dependent autophagy pathway by AMPK. A549 cells were incubated with MRGX for 48 h. (A) Insoluble cellular fractions were lysed directly with SDS buffer and equal amounts of cell lysate were subjected to western blot analysis with AMPK, p-AMPK (T172), Akt, p-Akt, mTOR, p-mTOR, 4EBP1, p-4EBP1, Ulk1 and p-Ulk1 antibodies. (B) MRGX activated AMPK and Akt phosphorylation compared to compound C-treated A549 cells. GAPDH was used as an internal standard. n.s., not significant. Data are expressed as means $\pm \mathrm{SD}$. ${ }^{*} \mathrm{P}<0.05,{ }^{* * *} \mathrm{P}<0.001$.

protective autophagy in cancer cells, MRGX induced autophagy via an AMPK-Akt pathway.

\section{Discussion}

The pharmacological actions of ginseng are attributed to ginsenosides (29). More than 60 ginsenosides have been isolated from American ginseng, and novel ginsenosides are still being discovered $(30,31)$. Steaming or heating American ginseng alters the ginsenoside profiles and increases the anticancer activity. The boiled water and butanol extracts of mountain ginseng exhibit strong anti-lung cancer activity, and ginsenoside profiling of these extracts has identified $\mathrm{Rg} 3$, $\mathrm{Rh} 2$ and compound $\mathrm{K}$ as being more prevalent than they are in regular ginseng (32). In the present study, we evaluated the anticancer activity of enzyme-treated regular ginseng root in lung cancer cells via autophagy activation. Treatment with MRGX at concentrations of $50-100 \mu \mathrm{g} / \mathrm{ml}$ for 48 and $72 \mathrm{~h}$ markedly and significantly inhibited the proliferation of A549 cells (Fig. 1). The inhibitory effect of MRGX was mediated through G2/M phase arrest (Fig. 2) and induction of autophagic cell death. To analyze MRGX treatment-mediated expression of autophagy-related genes in lung cancer cells, we used a cDNA microarray approach. The clustered microarray data identified groups of genes that were regulated differentially in the control and MRGX-treated cells. The genes whose expression levels were altered by at least 2-fold are shown in Fig. 3A. Three groups of genes (2-fold increased, 2-fold decreased and autophagy-related genes) are shown. Among these, 22 genes were increased by $>2$-fold, and 3 were decreased by $>2$-fold; autophagy-related genes are shown individually using Venn diagrams (Fig. 3B). The autophagy-related genes are listed in Table I. To explore the major autophagy-related proteins identified using the Gene Ontology analyses, we used IPA to query 25 proteins that were upregulated or downregulated by MRGX. These analyses resulted in a distinct interconnected network of 25 proteins (Fig. 4). Among these, Beclin-1, ATG5 and Ulk1 were the centers of the MRGX-related autophagy protein network.

Autophagy is a conserved catabolic process that depends not only on cellular stress response, but also on the recycling of protein and cellular organelles for cell death. Autophagy is initiated by the formation of a double-membrane vesicle called an autophagosome, which upon formation sequesters cellular components for delivery to the lysosome. Although it is not an ideal autophagy marker, acridine orange staining can be used to assess the autophagy status. In A549 cells treated with $0-100 \mu \mathrm{g} / \mathrm{ml}$ of MRGX, AVOs were quantified using acridine orange staining and flow cytometry. The number of AVOs were found to increase gradually in an MRGX concentrationdependent manner (Fig. 5). The autophagy process requires several different AuTophaGy (ATG) factors. Ulk1-ATG13 complex controls initiation, Beclin-1-class III PI3K is needed for nucleation, and microtubule-associated protein light chain 3 (LC3) regulates elongation. Autophagy can be monitored using microscopic and bio-chemical methods, and the LC3, Beclin-1 and ATG5 proteins are useful autophagy markers since they associate with the isolation membrane and spherical autophagosomes (33). The cellular localization of LC3, Beclin-1 and ATG5 can be easily visualized using a recombinant protein fused with a green fluorescent protein (GFP) (34). Punctate GFP-LC3, GFP-Beclin-1 and GFP-ATG5 spots in the cytoplasm reveal autophagosome formation (Fig. 6A and B), and the numbers of GFP-LC3, GFP-Beclin-1 and GFP-ATG5 spots represent the level of autophagy induction. Cytosolic LC3-I and autophagosome-bound LC3-II levels are established 
indicators of autophagy (35). Since transient overexpression of GFP-LC3 often results in false-positive outcomes, we used a GFP-LC3 stable cell line for testing. MRGX treatment resulted in cytoplasmic LC3 foci in the A549 cells and altered the expression of autophagy markers, specifically increasing the expression of LC3-II. The linker protein p62/SQSTM1 (p62) includes a ubiquitin-associated binding domain and an LC3-interacting region. In this way, p62 scavenges ubiquitinated proteins for degradation and binds LC3 to anchor these proteins into the forming autophagosome. Beclin-1 and ATG5 were also increasingly expressed cytoplasmically with lysosomes (Fig. 6C). Together, these data suggest that MRGX is, indeed, an autophagy inducer.

The mTOR pathway is an integral cell growth regulator involving TORC1 and TORC2, which have been defined by both their association with Raptor or Rictor, respectively, and their sensitivity to short-term rapamycin inhibition. AMPK is a ubiquitously expressed kinase that plays a key role in energy homeostasis. Stimulation of AMPK activates autophagy directly through phosphorylation of Ulk1, a key initiator of the autophagic process. The Akt pathway to mTOR signaling is a feature of control in mammalian autophagy caused by AMPK. In Fig. 7A, AMPK phosphorylation (T172) expression markedly increased the level of p-Akt. The increase in the Akt activity in A549 cells treated with a $100 \mu \mathrm{g} / \mathrm{ml}$ concentration of MRGX was correlated with a decrease in mTOR activity, as determined from 4EBP1 phosphorylation. 4EBP1 plays a prominent role in mediating the effects of these pathways in cancer cells. AMPK promoted autophagy by directly activating Ulk1 through phosphorylation of S317, but not S757. There are over 20 phosphorylation sites on Ser, and residue phosphorylation is essential for controlling its activity (36). Mechanistically, the level of p-AKT was increased, but that of p-mTOR was decreased, in the A549 cells treated with MRGX at a concentration of $100 \mu \mathrm{g} / \mathrm{ml}$ compared to the control. The downregulation of mTOR due to MRGX treatment depended on the levels of p-4EBP1 and p-Ulk (S317), which depend on the autophagy pathway caused by AMPK (T172). Active AMPK inhibits mTOR and 4EBP1 to ensure that cells maintain essential nutrients and energy during metabolic crises. Despite the importance of kinase, no specific chemical inhibitors are available to examine its function. However, compound $\mathrm{C}$ (dorsomorphin) has been widely used in cellular, biochemical, and in vivo assays as a selective AMPK inhibitor. When cells were treated with compound $\mathrm{C}$, phosphorylation of AMPK and Akt was decreased. However, following treatment with compound $\mathrm{C}$ and MRGX treatment, the p-AKT and the p-AMPK levels were increased (Fig. 7B), and Ulk1 phosphorylation induced autophagy. The ability of MRGX to inhibit mTOR signaling in an Akt-dependent manner and to induce autophagy signaling in an AMPK-Ulk1-dependent manner suggests the existence of a unique signaling pathway in these cells. Akt inhibited 4EBP1 phosphorylation through mTOR, which led to the activation of the TORC1 complex pathway whereas AMPK upregulated by MRGX activated LC3, Beclin-1 and ATG5. These results suggest that MRGX induces autophagy signaling by modulating AMPK-Ulk1 in A549 lung cancer cells.

In the present study, we showed activation of autophagy as a result of MRGX treatment. As excessive autophagy has potential to induce tumor-cell death, further study will be required to reveal the role of MRGX-mediated autophagy induction in lung cancer cell death.

\section{Acknowledgements}

The present study was supported by the Creative Fusion Research Program through the Creative Allied Project funded by the Korean Research Council for Fundamental Science and Technology (CAP-12-1-KIST), the Korea Basic Science Institute Research Program (C36955) and Korea Institute of Oriental Medicine (K16060).

\section{References}

1. Ang-Lee MK, Moss J and Yuan CS: Herbal medicines and perioperative care. JAMA 286: 208-216, 2001.

2. Nag SA, Qin JJ, Wang W, Wang MH, Wang H and Zhang R: Ginsenosides as anticancer agents: In vitro and in vivo activities, structure-activity relationships, and molecular mechanisms of action. Front Pharmacol 3: 25, 2012.

3. Kwon YK, Lee SY, Kang HS, Sung JS, Cho CK, Yoo HS, Shin S, Choi JS, Lee YW and Jang IS: Differential expression of gene profiles in MRGX-treated lung cancer. J Pharmacopuncture 16: 30-38, 2013.

4. Kim KH, Choi I, Lee YW, Cho CK, Yoo HS, Lee SB, Ho Choi S, Kwon KR and Jang JH: Target genes involved in antiproliferative effect of modified ginseng extracts in lung cancer A549 cells. Acta Biochim Biophys Sin 46: 441-449, 2014.

5. Jang SI, Lee YW, Cho CK, Yoo HS and Jang JH: Identification of target genes involved in the antiproliferative effect of enzyme-modified ginseng extract in HepG2 hepatocarcinoma cell. Evid Based Complement Alternat Med 2013: 502568, 2013.

6. Hwang JW, Baek YM, Jang IS, Yang KE, Lee DG, Yoon SJ, Rho J, Cho CK, Lee YW, Kwon KR, et al: An enzymatically fortified ginseng extract inhibits proliferation and induces apoptosis of KATO3 human gastric cancer cells via modulation of Bax, mTOR, PKB and IкB $\alpha$. Mol Med Rep 11: 670-676, 2015.

7. Stinchcombe TE, Lee CB and Socinski MA: Current approaches to advanced-stage non-small-cell lung cancer: First-line therapy in patients with a good functional status. Clin Lung Cancer 7 (Suppl 4): S111-S117, 2006.

8. Jemal A, Siegel R, Ward E, Hao Y, Xu J and Thun MJ: Cancer statistics, 2009. CA Cancer J Clin 59: 225-249, 2009.

9. Yun TK: Panax ginseng - a non-organ-specific cancer preventive? Lancet Oncol 2: 49-55, 2001.

10. Lee DG, Jang SI, Kim YR, Yang KE, Yoon SJ, Lee ZW, An HJ, Jang IS, Choi JS and Yoo HS: Anti-proliferative effects of ginsenosides extracted from mountain ginseng on lung cancer. Chin J Integr Med 22: 344-352, 2016.

11. Gustafsson AB and Gottlieb RA: Recycle or die: The role of autophagy in cardioprotection. J Mol Cell Cardiol 44: 654-661, 2008.

12. Kundu M and Thompson CB: Autophagy: Basic principles and relevance to disease. Annu Rev Pathol 3: 427-455, 2008.

13. Xie Z, Zhang J, Wu J, Viollet B and Zou MH: Upregulation of mitochondrial uncoupling protein-2 by the AMP-activated protein kinase in endothelial cells attenuates oxidative stress in diabetes. Diabetes 57: 3222-3230, 2008.

14. Colombo SL and Moncada S: AMPKalpha1 regulates the antioxidant status of vascular endothelial cells. Biochem J 421: 163-169, 2009.

15. Vellai T: Autophagy genes and ageing. Cell Death Differ 16: 94-102, 2009.

16. Stockert JC, Blázquez-Castro A, Cañete M, Horobin RW and Villanueva A: MTT assay for cell viability: Intracellular localization of the formazan product is in lipid droplets. Acta Histochem 114: 785-796, 2012.

17. Choi IK, Cho YS, Jung HJ and Kwon HJ: Autophagonizer, a novel synthetic small molecule, induces autophagic cell death. Biochem Biophys Res Commun 393: 849-854, 2010.

18. Hwang JW, Baek YM, Yang KE, Yoo HS, Cho CK, Lee YW, Park J, Eom CY, Lee ZW, Choi JS, et al: Lactobacillus casei extract induces apoptosis in gastric cancer by inhibiting NF- $\mathrm{KB}$ and mTOR-mediated signaling. Integr Cancer Ther 12: 165-173, 2013. 
19. Mizushima N and Yoshimori T: How to interpret LC3 immunoblotting. Autophagy 3: 542-545, 2007.

20. Hammond EM, Brunet CL, Johnson GD, Parkhill J, Milner AE, Brady G, Gregory CD and Grand RJ: Homology between a human apoptosis specific protein and the product of $A P G 5$, a gene involved in autophagy in yeast. FEBS Lett 425: 391-395, 1998.

21. Kang R, Zeh HJ, Lotze MT and Tang D: The Beclin 1 network regulates autophagy and apoptosis. Cell Death Differ 18: 571-580, 2011.

22. Cheong H, Wu J, Gonzales LK, Guttentag SH, Thompson CB and Lindsten T: Analysis of a lung defect in autophagy-deficient mouse strains. Autophagy 10: 45-56, 2014.

23. Traganos F and Darzynkiewicz Z: Lysosomal proton pump activity: Supravital cell staining with acridine orange differentiates leukocyte subpopulations. Methods Cell Biol 41: 185-194, 1994.

24. Yoon JH, Her S, Kim M, Jang IS and Park J: The expression of damage-regulated autophagy modulator 2 (DRAM2) contributes to autophagy induction. Mol Biol Rep 39: 1087-1093, 2012.

25. Liu J, Xia H, Kim M, Xu L, Li Y, Zhang L, Cai Y, Norberg HV, Zhang T, Furuya T, et al: Beclin1 controls the levels of p53 by regulating the deubiquitination activity of USP10 and USP13. Cell 147: 223-234, 2011.

26. Guévin C, Manna D, Bélanger C, Konan KV, Mak P and Labonté P: Autophagy protein ATG5 interacts transiently with the hepatitis C virus RNA polymerase (NS5B) early during infection. Virology 405: 1-7, 2010.

27. Egan D, Kim J, Shaw RJ and Guan KL: The autophagy initiating kinase ULK1 is regulated via opposing phosphorylation by AMPK and mTOR. Autophagy 7: 643-644, 2011.
28. Alers S, Löffler AS, Wesselborg S and Stork B: Role of AMPKmTOR-Ulk1/2 in the regulation of autophagy: Cross talk, shortcuts, and feedbacks. Mol Cell Biol 32: 2-11, 2012.

29. Yuan CS, Wu JA and Osinski J: Ginsenoside variability in American ginseng samples. Am J Clin Nutr 75: 600-601, 2002.

30. Lei J, Li X, Gong XJ and Zheng YN: Isolation, synthesis and structures of cytotoxic ginsenoside derivatives. Molecules 12: 2140-2150, 2007

31. Han M, Hou JG, Dong CM, Li W, Yu HL, Zheng YN and Chen L: Isolation, synthesis and structures of ginsenoside derivatives and their anti-tumor bioactivity. Molecules 15: 399-406, 2010.

32. Hwang JW, Oh JH, Yoo HS, Lee YW, Cho CK, Kwon KR, Yoon JH, Park J, Her S, Lee ZW, et al: Mountain ginseng extract exhibits anti-lung cancer activity by inhibiting the nuclear translocation of NF-кB. Am J Chin Med 40: 187-202, 2012.

33. Levine B and Klionsky DJ: Development by self-digestion: Molecular mechanisms and biological functions of autophagy. Dev Cell 6: 463-477, 2004.

34. Hu D, Wu J, Xu L, Zhang R and Chen L: A method for the establishment of a cell line with stable expression of the GFP-LC3 reporter protein. Mol Med Rep 6: 783-786, 2012.

35. Ding Z, Wang X, Schnackenberg L, Khaidakov M, Liu S, Singla S, Dai Y and Mehta JL: Regulation of autophagy and apoptosis in response to ox-LDL in vascular smooth muscle cells, and the modulatory effects of the microRNA hsa-let-7g. Int J Cardiol 168: 1378-1385, 2013.

36. Roach PJ: AMPK $\rightarrow$ ULK1 $\rightarrow$ autophagy. Mol Cell Biol 31: 3082-3084, 2011 